\title{
Gut hormones and appetite dysregulation in Crohn's disease
}

\author{
G. W. Moran ${ }^{1,2}$, F. Leslie ${ }^{2}$ and J. T. Mclaughlin ${ }^{1}$ \\ ${ }^{1}$ Epithelial Sciences, Stopford Building, University of Manchester, Oxford Road, Manchester M13 9PL, UK and \\ ${ }^{2}$ Gastroenterology Department, City General Hospital, University Hospital of North Staffordshire, Newcastle Road, \\ Stoke-on-Trent ST5 6QG, UK
}

Loss of appetite and malnutrition in active Crohn's disease (CD) are very important problems, and up to $7.2 \%$ of patients with $\mathrm{CD}$ are malnourished ${ }^{(1,2)}$. However, the biological mechanisms underpinning appetite loss are unclear. Enteroendocrine cells (EEC) constitute $\sim 1 \%$ of the intestinal lining mucosa and form a pivotal part of the brain-gut-axis that controls appetite and satiety. They secrete gut hormones such as glucagon-like peptide-1 (GLP-1) and polypeptide YY (PYY) which act on appetite control centres in the brainstem through an endocrine or paracrine pathway. Models of intestinal inflammation in mice have shown an up-regulation of EEC and their hormone products leading to hypophagia and weight loss ${ }^{(3)}$. Similar changes were shown in human infections with Giardia lamblia ${ }^{(4)}$. It is now essential to explore this novel mechanistic link to patients with chronic intestinal inflammation like CD.

Sixteen patients with active small bowel CD (SB-CD), 5 patients with active large bowel CD (LB-CD) and 12 healthy controls were recruited for this study. Disease activity was classified through independent histopathological/endoscopic assessments, validated patient questionnaires and biochemical scores. Gut hormone responses to a mixed nutrient test meal (Heinz Cream of Chicken Soup, $300 \mathrm{~g}$ ) were studied using a multiplex ELISA technique (Luminex). Patient symptoms were assessed using a validated visual analogue score (VAS) ${ }^{(4)}$. A subgroup of patients who achieved remission (without surgery or anti-TNF $\alpha$ treatment) was also re-studied later.

CD patients displayed a significant 6-fold reduction in appetite parameters by VAS $(P<0.0001)$ both pre- and post-prandially. Total PYY showed a 2-fold increase in post-prandial levels in the SB-CD group when compared with controls $(P=0.038)$. PYY was, however, not elevated in the LB-CD group. A significant correlation was observed between post-prandial PYY responses and symptoms, in SB-CD specifically nausea and bloatedness $(P=0.04$ and 0.03 , respectively). Basal GLP-1 values were similar between groups and increased post-prandially. However, the post-prandial levels of active GLP-1 remained persistently elevated over 60 min in the SB-CD group, while levels fell in controls $(P=0.01)$. The orexigenic hormone ghrelin was basally 3 -fold higher in the $\mathrm{CD}$ group and, rather than showing normal physiological suppression by the meal, ghrelin levels showed a paradoxical positive postprandial response and a significant correlation with disease activity $(P=0.02)$.

In remission, the VAS reverted to those of normal controls. Ghrelin also returned to a normal negative post-prandial response in remission. The sustained elevation in post-prandial GLP-1 level in active disease also normalised. Total PYY, however, showed no change compared to active disease.

These results are compatible with a potential role of EEC in appetite dysregulation in intestinal inflammation. Enhanced EEC responses may adversely affect appetite in such patients through increased gut-brain signalling before and after eating, and provide novel therapeutic targets. Further work is underway to further dissect the neuroendocrine circuitry in this system.

Supported by Mavis Rushton Bequest Fund, University Hospital of North Staffordshire.

1. Nguyen GC, Munsel M \& Harris M (2008) Inf Bowel Dis 14, 1105-1111.

2. Valentini L, Schaper L, Buning C et al. (2008) Nutrition 24, 694-702.

3. McDermott JR, Leslie F, D’Amato M et al. (2006) Gut 55, 492-497.

4. Leslie FC, Thompson D, McLaughlin J et al. (2003) Q J Med 96, 870-871. 